\title{
Olfactory Dysfunction and Its Relationship with Clinical Symptoms of Alzheimer Disease
}

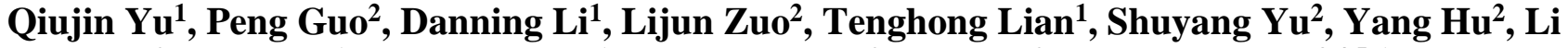 \\ Liu $^{2}$, Zhao Jin ${ }^{1}$, Ruidan Wang1, Yingshan Piao², Lixia Li², Xiaomin Wang ${ }^{3,4,5,6}$, Wei \\ Zhang1,2,4,5,6,7,*
}

\begin{abstract}
${ }^{1}$ Department of Neurology, Beijing Tiantan Hospital, Capital Medical University, Beijing, 100050, China. ${ }^{2}$ Department of Geriatrics, Beijing Tiantan Hospital, Capital Medical University, Beijing, 100050, China. ${ }^{3}$ Department of Physiology, Capital Medical University, Beijing, 100069, China. ${ }^{4}$ Key Laboratory for Neurodegenerative Disorders of the Ministry of Education, Capital Medical University, Beijing, 100069, China. ${ }^{5}$ Center of Parkinson Disease, Beijing Institute for Brain Disorders, Beijing, 100069, China. ${ }^{6}$ Beijing Key Laboratory on Parkinson Disease, Beijing, 100053, China. ${ }^{7}$ China National Clinical Research Center for Neurological Diseases, Beijing, 100050, China
\end{abstract}

[Received May 12, 2018; Revised August 1, 2018; Accepted August 19, 2018]

\begin{abstract}
Our study aimed to analyse the olfactory dysfunction (OD) evaluations between self-report, the Hyposmia Rating Scale (HRS) and the Sniffin' Sticks test, and the relationship between OD and clinical features of AD. Sixty patients with AD dementia, 37 patients with mild cognitive impairment (MCI) due to AD and 30 healthy controls were consecutively recruited. Olfactory function was evaluated by self-report, HRS and Sniffin' Sticks test. Patients were divided into AD with OD (AD-OD) and AD with no OD (AD-NOD) groups based on the results of the Sniffin' Sticks test. Cognitive symptoms and neuropsychiatric symptoms were assessed by corresponding scales, and activities of daily living (ADL) were assessed by the ADL scale. In the control, MCI due to $\mathrm{AD}$ and $\mathrm{AD}$ dementia groups, the frequency of $\mathrm{OD}$ was $10.0 \%, 13.5 \%$ and $18.3 \%$, respectively, by selfreport; $6.7 \%, 24.3 \%$ and $48.3 \%$, respectively, by HRS; and $3.3 \%, 13.5 \%$ and $65.0 \%$, respectively, by the Sniffin' Sticks test. Compared to the results of the Sniffin' Sticks test, the diagnostic coincidence rates of OD by HRS in patients with MCI due to AD and AD dementia were $89.2 \%$ and $66.7 \%$, respectively.Compared to the AD-NOD group, the scores of global cognition and memory, visuospatial ability and attention were all decreased $(P<0.05)$, the apathy score was increased $(P<0.05)$, and the ADL score was elevated $(P<0.01)$. The frequency and accuracy of OD by self-report is relatively low. HRS can be used for screening olfaction in patients with MCI due to AD. The Sniffin' Sticks test can be used for validating OD in AD patients. AD-OD patients have severe impairments in global cognition and multiple cognitive domains of memory, visuospatial ability and attention, as well as neuropsychiatric symptoms of apathy, and thus have seriously compromised ADL.
\end{abstract}

Key words: Alzheimer disease, olfactory dysfunction, self-report, Hyposmia Rating Scale, Sniffin' Sticks test, clinical features

Alzheimer disease (AD) is a progressive neurodegenerative disease and is the commonest form of dementia in the elderly population. The incidence and prevalence of $\mathrm{AD}$ are increasing with the rapid aging of the population. Great attention has been paid to cognitive impairment, neuropsychiatric symptoms and activities of daily living (ADL) for $\mathrm{AD}$ patients. In recent years, it was found that olfactory dysfunction (OD) occurred in $\mathrm{AD}$ patients, even in those who were at an early stage of disease [1]. OD might predict the progression of AD [2].

*Correspondence should be addressed to: Dr. Wei Zhang, Department of Neurology, Department of Geriatrics, Beijing Tiantan Hospital, Capital Medical University. Beijing, China. Email: ttyyzw@163.com

Copyright: ( 2018 Yu Q et al. This is an open-access article distributed under the terms of the Creative Commons Attribution License, which permits unrestricted use, distribution, and reproduction in any medium, provided the original author and source are credited. 
However, OD has been largely ignored by both doctors and patients despite its early appearance in $\mathrm{AD}$ [3].

OD includes disturbances in olfactory threshold, discrimination, and identification. The majority of studies have only examined olfactory identification in patients with mild cognitive impairment (MCI) due to AD [4] or dementia due to $\mathrm{AD}$ (AD dementia) [5]. In a few studies, olfactory functions other than identification have been examined. Only a handful of them included more than one olfactory test, and most often these were olfactory threshold and identification [6]. However, in addition to patients with MCI due to $\mathrm{AD}$ and patients with $\mathrm{AD}$ dementia, some proportion of the normal population has OD. We therefore conducted the present study to evaluate three aspects of olfactory function, including olfactory threshold, discrimination, and identification, in control, $\mathrm{MCI}$ due to $\mathrm{AD}$ and $\mathrm{AD}$ dementia groups.

OD is largely ignored by patients, so we first asked each subject whether he or she had OD according to their perception of odor. Then, we used the Hyposmia Rating Scale (HRS), which might offer a simple and time-saving approach to screening OD. OD can be validated by objective and reliable tests, such as the University of Pennsylvania Smell Identification Test (UPSIT) [7] and Sniffin' Sticks test [8]. However, UPSIT is just used to assess olfactory identification, whereas the Sniffin' Sticks test comprehensively evaluates olfactory threshold, discrimination, and identification. We therefore determined the frequency of $\mathrm{OD}$ and investigated the relationship between OD and clinical symptoms of AD by the Sniffin' Sticks test in the present study.

Studies about AD with OD (AD-OD) are limited. Most of them have focused on the manifestations of ADOD [9] and the prediction of the conversion of MCI to AD $[10,11]$. However, there are few studies on the relationship between $\mathrm{OD}$ and clinical symptoms of $\mathrm{AD}$, including cognitive impairment, neuropsychiatric symptoms and ADL. A previous study found that olfactory identification worsened as the AD deteriorated, indicating its potential as a clinical marker of $\mathrm{AD}$ progression [2]. However, data from another study did not support this point of view [10]. Olfactory discrimination is related to executive function and semantic memory [12], but there has been no investigation of the correlation of AD-OD with other cognitive domains, such as attention, language and visuospatial ability. Moreover, few studies on the relationship between AD-OD and neuropsychiatric symptoms, such as apathy, anxiety, depression and agitation, have been conducted.

In this study, in patients with MCI due to AD, patients with $\mathrm{AD}$ dementia, and control subjects, $\mathrm{OD}$ was investigated by self-report, HRS and the Sniffin' Sticks test to evaluate the efficacy and clinical application of the three approaches of OD evaluation. Clinical features of
AD-OD were investigated by analyzing demographic variables and the scores of rating scales for clinical symptoms of cognition, neuropsychiatric symptoms and ADL with the aim of determining the relationship between $\mathrm{OD}$ and $\mathrm{AD}$.

\section{MATRIALS AND METHODS}

\section{Ethics statement}

This study met the guidelines of Helsinki Declaration on ethical principles for medical research involving human subjects, and the protocol was approved by the ethical review board of Beijing Tiantan Hospital. All participants signed written informed consents before they were recruited in the study. All methods were performed in accordance with the relevant guidelines and regulations.

\section{Patients with $A D$}

Inclusion criteria: This study included patients with MCI due to $\mathrm{AD}$ [13] and patients with $\mathrm{AD}$ dementia [14] according to National Institute of Aging and Alzheimer's Association (NIA-AA) criteria.

Exclusion criteria: (1) acute respiratory infections within 3 weeks; (2) chronic nasitis and sinusitis, and chronic obstructive pulmonary disease; (3) long-term or significant exposure to volatile substances, such as pesticides, herbicides, metallic dusts, acid fumes, industrial solvents, cleaning products or sawdust; (4) severe head trauma, nasal surgery; (5) smoking and drug abuse; (6) other neuropsychiatric disorders affecting olfactory function, such as Parkinson disease, multiple sclerosis and epilepsy.

Total 97 AD patients were consecutively recruited from the Departments of Geriatrics and Neurology, Beijing Tiantan Hospital, Capital Medical University, from November 2014 to March 2017. Of the 97 AD patients, 37 were diagnosed with $\mathrm{MCI}$ due to $\mathrm{AD}$ and 60 had AD dementia.

\section{Control participants}

Thirty healthy controls from the community were recruited based on the following criteria: (1) no cognitive impairment; (2) no intracranial diseases, including encephalitis, meningitis, cerebrovascular disease, epilepsy and tumors; (3) no psychosis or dysarthria that affected expression; (4) no acute respiratory infections within the previous 3 weeks; (5) no chronic nasitis or sinusitis or chronic obstructive pulmonary disease; (6) no long-term or significant exposure to volatile substances, such as pesticides, herbicides, metallic dusts, acid fumes, industrial solvents, cleaning products and sawdust; (7) no 
severe head trauma or nasal surgery; (8) no cigarette smoking or drug abuse; (9) no other neuropsychiatric disorders affecting olfactory function, such as Parkinson disease, multiple sclerosis and epilepsy.

\section{Collection of demographic information}

Demographic variables, including gender, age, age of onset, disease duration, education level, and smoking were recorded for all $\mathrm{AD}$ participants. Demographic variables, including gender, age, education level, and smoking were recorded for control participants.

\section{Evaluation of olfactory function by Sniffin' Sticks test}

Olfactory function was evaluated for each participant by the Sniffin' Sticks test. Sniffin' Sticks were purchased from Burghart Messtenik Company (Tinsdaler Weg 175 22880 Wedel Germany; product number: LA-13-00005). There were a total 112 sticks, in which 48 were for testing olfactory threshold (THR), 48 for testing olfactory discrimination (DIS) and 16 for testing olfactory identification (ID).

Participants were exposed to n-butanol samples from the lowest to the highest concentration. In the olfactory THR test, the score of THR was the ranking of the Sniffin' Sticks when participants could identify the minimal concentration of n-butanol. The lower the THR score, the worse the function of recognition.

In the olfactory DIS test, participants were instructed to name the target odor that was different from the other two odors. The score of DIS was the number of Sniffin' Sticks that participants correctly answered. The lower the DIS score, the worse the function of discrimination.

In the olfactory ID test, participants were required to choose the name of the odor he or she smelled from 4 given choices. The score of ID was the number of Sniffin' Sticks that participants correctly answered. The lower the ID score, the worse the function of identification.

Overall olfactory function was assessed by summing up the scores of THR, DIS and ID, which was abbreviated as TDI.

Olfactory function was identified by the following criteria deriving from a cross-sectional study of olfactory function in 3282 people by the Sniffin' Sticks test adjusted for sex and gender [8]. OD was diagnosed in males aged $36-55$ years with TDI score $\leq 24$ points, in females aged $36-55$ years with TDI score $\leq 28$ points, and in males or females aged $>55$ years, with TDI score $\leq 19$ points. No OD was diagnosed in males aged 36-55 years with TDI score $\geq 25$ points, in females aged $36-55$ years with TDI score $\geq 29$ points, or in males or females aged $>55$ years with TDI score $\geq 20$ points.

\section{Screening of olfactory function by HRS}

HRS was validated by the Sniffin' Sticks test in PD patients by Millar in 2012 [15] and was used to evaluate olfactory function in AD patients and control participants in this study. HRS includes 6 items, each one describing the level of olfactory function from 0 to 4 point (s), for a total score of 24 points. Total HRS score was obtained by summing up the score of each item. The higher the score of HRS, the better the function of olfaction. The optimal cut-off value for HRS was 22.5 points, with a sensitivity of $70 \%$ and a specificity of $85 \%$ [15]. Thus, AD patients with total HRS score $\geq 23$ points and $\leq 22$ points were defined as with OD and with no OD, respectively [15].

The sensitivity, specificity, positive predictive value and negative predictive value of HRS were calculated with reference to the results of the Sniffin' Sticks test. Sensitivity $=$ true positive/(true positive + false negative) $\mathrm{x} 100 \%$, specificity $=$ true negative $/$ (true negative + false positive) $\mathrm{x} 100 \%$, positive predictive value $=$ true positive/(true positive + false positive) $\times 100 \%$, negative predictive value $=$ true negative/(true negative + false negative $) \times 100 \%$, and diagnostic accordance rate $=($ true positive + true negative)/total $\times 100 \%$. Sensitivity reflects the ability to diagnose OD by HRS. Specificity reflects the ability to judge people who actually have NOD by HRS. Positive predictive value refers to how many people diagnosed with OD truly have OD. Negative predictive value refers to how many people diagnosed with NOD truly have NOD. The diagnostic accordance rate shows the degree of agreement between HRS and the Sniffin' Sticks test and reflects exactly the ability to diagnosis OD and NOD.

\section{Assessments of clinical symptoms of $A D$}

\section{Cognitive function: overall cognitive function}

The Mini-Mental State Examination (MMSE) and Montreal Cognitive Assessment (MoCA) were used to rate the overall cognitive function of $\mathrm{AD}$ patients. Patients with illiteracy, primary education, or more than a junior education were identified as having cognitive impairment when the MMSE score was below 17, 20 or 24 points, respectively. MoCA score $\leq 26$ indicated potential cognitive impairment. If the educational level of an individual was less than 12 years, 1 point was added. The lower the scores of the two scales, the severer the cognitive impairment.

\section{Individual cognitive domain}

Individual cognitive domain was assessed by using a variety of rating scales, as follows:

Memory: The Auditory Verbal Learning Test [16, 17] (AVLT) was used to assess verbal memory. AVLT1-3, 
AVLT4 and AVLT5 stand for immediate recall, shortdelayed recall and long-delayed recall, respectively. The total recall indicated by the first 5 times taking the AVLT reflects the general state of verbal memory.

The Complex Figure Test [17] (CFT)-delayed memory was used to assess visual delayed memory.

The lower the scores of the above scales, the worse the memory of the participant.

\section{Visuospatial ability}

CFT [17]-imitation was used to evaluate visuospatial ability. A lower score represented the worse visuospatial ability.

\section{Language function}

The Animal Fluency Test [18] (AFT) was used to assess language function. If the score was lower, the language function was poorer.

\section{Attention}

The Trail Making Test A [19] (TMT-A) was used to evaluate attention. The longer the time spent, the worse the patient's attention was.

The Symbol Digit Modalities Test [20] (SDMT) was also used to assess attention. The lower the SDMT score, the worse the attention of the subject.

\section{Executive function}

The Stroop Color-Word Test [19] (SCWT) was used to evaluate executive function. The lower the score was, the worse the executive function was.

The Trail Making Test B [19] (TMT-B) was also used to assess executive function. The longer the time consumed, the worse the executive function that the patients had.

\section{Neuropsychiatric symptoms}

\section{Overall neuropsychiatric symptoms}

The Neuropsychiatric Inventory (NPI) was used to assess the overall neuropsychiatric symptoms of $\mathrm{AD}$ patients. The higher the NPI score was, the worse the overall neuropsychiatric symptoms were.

\section{Individual neuropsychiatric symptom}

Individual neuropsychiatric manifestation was then assessed by using a body of rating scales:

(1) Depression was assessed by the Hamilton Depression Scale (HAMD)-24 items. The higher the score was, the severer the depression was. The score $>8$ points suggested depression.

(2) Anxiety was assessed by the Hamilton Anxiety Scale (HAMA)-14 items. The higher the score was, the severer the anxiety was. The score $>8$ points indicated anxiety.

(3) Agitation was assessed by the Cohen-Mansfield Agitation Inventory (CMAI). The higher the CMAI score, the worse the agitation of the participant.

(4) Apathy was assessed by the Modified Apathy Estimate Scale (MAES). The higher the score was, the severer the apathy was. The score $>14$ points reflected clinically meaningful apathy.

Activities of daily living (ADL)

The ADL scale includes basic ADL (BADL) and instrumental ADL (IADL), which were assessed by the Katz basic ADL scale [21] and the Lawton and Brody instrumental ADL scale [22], respectively. The higher the score, the worse the ADL.

Table 1. Demographic variables among groups of control, MCI due to AD and AD dementia.

\begin{tabular}{|c|c|c|c|c|c|}
\hline Demographic variables & $\begin{array}{l}\text { Control group } \\
\text { (30 cases) }\end{array}$ & $\begin{array}{l}\text { MCI due to AD group } \\
\text { (37 cases) }\end{array}$ & $\begin{array}{l}\text { AD dementia group } \\
\text { (60 cases) }\end{array}$ & $P 1$ & $P 2$ \\
\hline$\overline{\text { Male/total [cases/total (\%)] }}$ & $10 / 30(33.33)$ & $10 / 37(27.03)$ & $24 / 60(40.00)$ & 0.575 & 0.193 \\
\hline Age [years, median (quartile)] & $62.00(60.00,69.50)$ & $64.00(58.50,71.00)$ & $71.00(62.00,78.00)$ & 0.529 & 0.019 \\
\hline $\begin{array}{l}\text { Age of onset } \\
\text { [years, median (quartile)] }\end{array}$ & & $60.00(54.75,70.00)$ & $67.50(58.75,74.25)$ & & 0.022 \\
\hline $\begin{array}{l}\text { Disease duration } \\
\text { [years, median (quartile)] }\end{array}$ & & $3.00(1.75,5.00)$ & $3.00(1.38,5.00)$ & & 0.559 \\
\hline Education [cases/total (\%)] & & & & 0.098 & 0.846 \\
\hline Primary school and below & $1 / 30(3.33)$ & $7 / 37(18.92)$ & $14 / 60(23.33)$ & & \\
\hline Middle and high school & $16 / 30(53.33)$ & $20 / 37(54.05)$ & $32 / 60(53.33)$ & & \\
\hline Bachelor's degree and above & $13 / 30(43.33)$ & $10 / 37(27.03)$ & $14 / 60(23.33)$ & & \\
\hline Smoking [cases/total (\%)] & $6 / 30(20.00)$ & $6 / 37(16.22)$ & $14 / 60(23.33)$ & 0.688 & 0.400 \\
\hline
\end{tabular}

P1: Control group vs MCI due to AD group

$P 2$ : MCI due to $\mathrm{AD}$ group vs $\mathrm{AD}$ dementia group 
Table 2. Comparisons of self-report, Hyposmia Rating Scale and Sniffin' Sticks test in the evaluation of OD among groups of Control, MCI due to AD and AD dementia.

\begin{tabular}{llll}
\hline & $\begin{array}{l}\text { Control group } \\
\text { (30 cases) }\end{array}$ & $\begin{array}{l}\text { MCI due to AD group } \\
\text { (37 cases) }\end{array}$ & $\begin{array}{l}\text { AD dementia group } \\
(\mathbf{6 0} \text { cases) }\end{array}$ \\
\hline Self-report [cases/total (\%)] & $3 / 30(10.0)$ & $5 / 37(13.5)$ & $11 / 60(18.3)$ \\
Hyposmia Rating Scale [cases/total (\%)] & $2 / 30(6.7)$ & $9 / 37(24.3)$ & $29 / 60(48.3)$ \\
Sniffin' Sticks test [cases/total (\%)] & $1 / 30(3.3)$ & $5 / 37(13.5)$ & $39 / 60(65.0)$ \\
\hline
\end{tabular}

\section{Data analyses}

Statistical analyses were performed by SPSS Statistics 20.0 (IBM Corporation, New York, USA). $P$ values < 0.05 were considered significant.

Continuous variables, if they were normally distributed, are presented as the means \pm standard deviations and were compared by the two-sample t test. Continuous variables, if they were not normally distributed, are presented as median (quartile) and were compared by a nonparametric test. Discrete variables were compared by the chi-square test.
Demographic variables and the scores of olfactory functions were compared among the control, MCI due to $\mathrm{AD}$ and $\mathrm{AD}$ dementia groups.

The scores of olfactory functions, including THR, DIS, ID and TDI, and clinical symptoms, including cognitive symptoms, neuropsychiatric symptoms and $\mathrm{ADL}$ as determined by the corresponding rating scales, were compared between AD-OD and AD-NOD groups.

Spearman correlation analyses were performed between the scores of THR, DIS, ID, TDI and the scores of cognitive symptoms, neuropsychiatric symptoms and $\mathrm{ADL}$ in the $\mathrm{AD}$ group.

Table 3. Comparisons of olfactory function among groups of Control, MCI due to AD and AD dementia by Sniffin' Sticks test.

\begin{tabular}{|c|c|c|c|c|c|c|}
\hline Olfactory variables & $\begin{array}{l}\text { Control group } \\
\text { (30 cases) }\end{array}$ & $\begin{array}{l}\text { MCI due to AD group } \\
\text { (37 cases) }\end{array}$ & $\begin{array}{l}\text { AD dementia group } \\
\text { (60 cases) }\end{array}$ & $P 1$ & $P 2$ & Adjusted $P 2$ \\
\hline TDI [points, median (quartile)] & $29.50(24.75,31.00)$ & $26.00(22.00,29.00)$ & $17.00(12.00,22.00)$ & 0.000 & 0.000 & 0.000 \\
\hline THR [points, median (quartile)] & $6.00(5.75,7.00)$ & $6.00(4.00,6.00)$ & $4.00(2.00,6.00)$ & 0.000 & 0.000 & 0.012 \\
\hline DIS [points, median (quartile)] & $11.50(8.75,12.00)$ & $10.00(8.00,11.00)$ & $6.50(4.00,8.00)$ & 0.001 & 0.000 & 0.000 \\
\hline ID [points, median (quartile)] & $12.00(10.00,13.00)$ & $10.00(8.00,13.00)$ & $7.00(5.00,9.00)$ & 0.055 & 0.000 & 0.000 \\
\hline
\end{tabular}

HRS: Hyposmia Rating Scale, TDI: Threshold + Discrimination + Identification, THR: Threshold, DIS: Discrimination, ID: Identification, $P 1$ : Control group vs $\mathrm{MCI}$ due to $\mathrm{AD}$ group, $P 2 \mathrm{MCI}$ due to $\mathrm{AD}$ group vs $\mathrm{AD}$ dementia group

\section{RESULTS}

\section{Comparisons of demographic variables among control, $M C I$ due to $A D$ and $A D$ dementia groups}

First, demographic variables, including gender, age, educational level, and smoking rate, were compared between the control group and the MCI due to AD group (Table 1). No significant differences in demographic variables were found between the two groups $(P>0.05)$.

Second, demographic variables were compared between the MCI due to $\mathrm{AD}$ group and the $\mathrm{AD}$ dementia group (Table 1). The age and age of onset in the $\mathrm{AD}$ dementia group were significantly older than those in the $\mathrm{MCI}$ due to $\mathrm{AD}$ group $(P<0.05)$. No significant difference was observed in other demographic variables between the two groups $(P>0.05)$.
Comparisons of self-report, HRS and Sniffin' Sticks test in the evaluation of $O D$ among control, MCI due to $A D$ and $A D$ dementia groups

(1) Self-report

Each subject was asked to report whether he or she had hyposmia. The frequency of OD in the control, MCI due to $\mathrm{AD}$ and $\mathrm{AD}$ dementia groups by self-report was $10.0 \%, 13.5 \%$ and $18.3 \%$, respectively (Table 2 ).

Among the 30 normal controls, $3(10.0 \%)$ reported OD, but none had OD by the Sniffin' Sticks test.

Of the 37 patients with MCI due to AD, 5 (13.5\%) reported OD, among which only $1(20.0 \%)$ had OD and 4 $(80.0 \%)$ did not by the Sniffin' Sticks test. 
Table 4. Comparison of Olfactory function between AD-NOD and AD-OD groups.

\begin{tabular}{llcc}
\hline Olfactory variables & $\begin{array}{c}\text { AD-NOD group } \\
\text { (53 cases) }\end{array}$ & $\begin{array}{c}\text { AD-OD group } \\
\text { (44 cases) }\end{array}$ & $\boldsymbol{P}$ \\
\hline TDI [points, median (quartile)] & $25.00(22.00,28.50)$ & $14.00(10.25,17.00)$ & $\mathbf{0 . 0 0 0}^{* *}$ \\
THR [points, median (quartile)] & $6.00(4.00,6.00)$ & $3.00(1.00,5.00)$ & $\mathbf{0 . 0 0 0}^{* *}$ \\
DIS [points, median (quartile)] & $10.00(8.00,11.00)$ & $5.00(3.00,7.00)$ & $\mathbf{0 . 0 0 0}^{* *}$ \\
ID [points, median (quartile)] & $10.00(9.00,12.50)$ & $6.00(3.00,8.00)$ & $\mathbf{0 . 0 0 0}^{* *}$ \\
\hline
\end{tabular}

TDI: Threshold + Discrimination + Identification, THR: Threshold, DIS: Discrimination, ID: Identification

Of the 60 patients with $\mathrm{AD}$ dementia, $11(18.3 \%)$ reported OD, among which $8(72.7 \%)$ had OD and 3 $(27.3 \%)$ did not by the Sniffin' Sticks test.

\section{(2) HRS}

HRS is a scale for screening olfactory function. In this study, the frequency of OD in the control, MCI due to AD and $\mathrm{AD}$ dementia groups by HRS was $6.7 \%, 24.3 \%$ and $48.3 \%$, respectively (Table 2 ).

Of the 30 subjects in the normal control group, 2 (6.7\%) had OD by HRS, but nobody was demonstrated to have OD by the Sniffin' Sticks test.
Of the 37 patients with MCI due to AD, 9 (24.3\%) had OD by HRS, but only $5(13.5 \%)$ had OD by the Sniffin' Sticks test. Of the remaining 28 patients $(75.7 \%)$ who did not present OD by HRS, nobody had OD by the Sniffin' Sticks test. Taking the Sniffin' Sticks test as the reference, the sensitivity, specificity, positive predictive value and negative predictive value of HRS were $100.0 \%$, $87.5 \%, 55.6 \%$ and $100.0 \%$, respectively. The diagnostic coincidence rate of OD by HRS in patients with MCI due to $\mathrm{AD}$ was $89.2 \%$.

Table 5. Comparison of cognitive function between AD-NOD and AD-OD groups.

\begin{tabular}{|c|c|c|c|}
\hline Cognitive variables & $\begin{array}{l}\text { AD-NOD group } \\
\text { (53 cases) }\end{array}$ & $\begin{array}{l}\text { AD-OD group } \\
\text { (4 cases) }\end{array}$ & $\bar{P}$ \\
\hline \multicolumn{4}{|l|}{ Global cognitive function } \\
\hline MMSE [points, median (quartile)] & $25.00(23.00,27.50)$ & $18.00(11.00,24.00)$ & $0.000^{* *}$ \\
\hline MoCA [points, median (quartile)] & $21.00(16.00,25.00)$ & $14.00(7.00,19.00)$ & $0.000^{* *}$ \\
\hline \multicolumn{4}{|l|}{ Memory } \\
\hline $\begin{array}{l}\text { AVLT-long delayed recall } \\
\text { [points, median (quartile)] }\end{array}$ & $3.00(0.00,5.75)$ & $0.00(0.00,0.25)$ & $0.001^{* *}$ \\
\hline $\begin{array}{l}\text { AVLT- the first } 5 \text { times total recall } \\
\text { [points, median (quartile)] }\end{array}$ & $16.50(10.00,26.75)$ & $10.00(8.00,13.25)$ & $0.001^{* *}$ \\
\hline $\begin{array}{l}\text { CFT- delayed memory } \\
\text { [points, median (quartile)] }\end{array}$ & $5.50(0.00,10.00)$ & $0.00(0.00,2.00)$ & $0.001^{* *}$ \\
\hline \multicolumn{4}{|l|}{ Language } \\
\hline AFT (points, mean \pm SD) & $15.54 \pm 4.61$ & $10.64 \pm 4.20$ & 0.151 \\
\hline \multicolumn{4}{|l|}{ Attention } \\
\hline $\begin{array}{l}\text { SDMT } \\
\text { [points, median (quartile)] }\end{array}$ & $22.00(18.00,32.00)$ & $16.00(8.25,23.00)$ & $\begin{array}{l}0.009^{* *} \\
0.000^{* *}\end{array}$ \\
\hline $\begin{array}{l}\text { TMT-A-time consuming } \\
\text { [seconds, median (quartile)] }\end{array}$ & $78.00(60.00,108.00)$ & $142.50(83.25,221.00)$ & \\
\hline Visual spatial function & & & $0.002^{* * *}$ \\
\hline $\begin{array}{l}\text { CFT- imitation } \\
\text { [points, median (quartile)] }\end{array}$ & $28.00(18.00,34.00)$ & $8.00(2.00,28.00)$ & \\
\hline \multicolumn{4}{|l|}{ Executive function } \\
\hline $\begin{array}{l}\text { SCWT- time consuming } \\
\text { [seconds, median (quartile)] }\end{array}$ & $94.00(72.50,120.25)$ & $127.00(76.50,176.75)$ & 0.059 \\
\hline $\begin{array}{l}\text { SCWT- correct number } \\
\text { [points, median (quartile)] }\end{array}$ & $48.00(43.75,50.00)$ & $44.00(39.25,50.00)$ & 0.124 \\
\hline $\begin{array}{l}\text { TMT-B- time consuming } \\
\text { [seconds, median (quartile)] }\end{array}$ & $238.00(195.00,240.00)$ & $240.00(167.00,240.00)$ & 0.695 \\
\hline
\end{tabular}

**: $P<0.01$. MMSE: Mini-Mental State Examination, MoCA: Montreal Cognitive Assessment, AVLT: Auditory Verbal Learning Test CFT: Complex Figure Test, AFT: Animal Fluency Test, SDMT: Symbol Digit Modalities Test, TMT: Trial Making Test, SCWT: Stroop Color Word Test 
Of the 60 patients with AD dementia, $29(48.3 \%)$ had OD by HRS, 24 (40.0\%) were demonstrated to have OD by the Sniffin' Sticks test. In the remaining 31 patients (51.7\%) who did not display OD by HRS, 15 had OD by the Sniffin' Sticks test. Compared with the results from the Sniffin' Sticks test, the sensitivity, specificity, positive predictive value and negative predictive value of HRS were $61.5 \%, 76.2 \%, 82.8 \%$ and $51.6 \%$, respectively. The diagnostic coincidence rate of OD by HRS in patients with $\mathrm{AD}$ dementia was $66.7 \%$.

(3) Sniffin' Sticks test

The Sniffin' Sticks test was adopted to objectively and comprehensively evaluate olfactory function. The frequency of $\mathrm{OD}$ in the control, $\mathrm{MCI}$ due to $\mathrm{AD}$ and $\mathrm{AD}$ dementia groups by the Sniffin' Sticks test was 3.3\%, $13.5 \%$ and $65.0 \%$, respectively (Table 2 ).

The frequency of AD-OD was determined by the results of the Sniffin' Sticks test. Of the 97 total AD patients, $44(45.4 \%)$ were demonstrated to have OD by the Sniffin' Sticks test. Thus, the frequency of AD-OD was $45.4 \%$.

Forty-four (45.4\%) and 53 patients $(54.6 \%)$ were divided into the $\mathrm{AD}$ with $\mathrm{OD}(\mathrm{AD}-\mathrm{OD})$ and $\mathrm{AD}$ with no OD (AD-NOD) groups, respectively.

\section{Comparisons of olfactory function among control, MCI due to $A D$ and $A D$ dementia groups}

(1) Comparisons of olfactory function between control and MCI due to AD groups

Comparison of olfactory function revealed that the scores of TDI, THR and DIS in the MCI due to AD group were all lower than those in the control group $(P<0.01)$ (Table 3). However, ID score was not significantly different between the two groups $(P>0.05)$.

(2) Comparison of olfactory function between MCI due to $\mathrm{AD}$ and $\mathrm{AD}$ dementia groups

Comparison of olfactory function indicated that the scores of TDI, THR, DIS and ID in the AD dementia group were lower than those in the MCI due to $\mathrm{AD}$ group $(P<0.01)$ (Table 3). After adjusting for age and age of onset, the scores of TDI, THR, DIS and ID in the AD dementia group were still significantly lower than those in the MCI due to AD group $(P<0.05)$ (Table 3$)$.

\section{Clinical evaluation of olfactory function between AD- $O D$ and $A D-N O D$ groups}

First, in the $97 \mathrm{AD}$ patients, the median scores of TDI, THR, DIS and ID were $21.00(15.00,26.00), 4.00$ (3.00, $6.00), 8.00(5.00,10.00)$ and $8.00(6.00,11.00)$ points, respectively. Further comparison revealed that the median scores of TDI, THR, DIS and ID in the AD-OD group were all significantly lower than those of the AD-NOD group $(P<0.01)$ (Table 4$)$.

\section{Relationships between olfactory function and clinical symptoms of $A D$}

(1) Relationships between the scores of olfactory function and cognitive symptoms

MMSE score in the AD-OD group was decreased compared with the AD-NOD group $(P<0.01)$ (Table 5). MMSE score was positively correlated with the scores of TDI, THR, DIS and ID $(P<0.05)$ (Supplemental table 1$)$.

MoCA score in the AD-OD group was reduced compared with the AD-NOD group $(P<0.01)$ (Table 5). MoCA score was positively correlated with the scores of TDI, THR, DIS and ID $(P<0.05)$ (Supplemental table 1$)$.

The score of AVLT-delayed memory in the AD-OD group was decreased compared with the AD-NOD group $(P<0.01)$ (Table 5). AVLT-delayed memory score was positively correlated with the scores of TDI, THR, DIS and ID $(P<0.05)$ (Supplemental table 2).

The score of total AVLT in the AD-OD group was reduced compared with the AD-NOD group $(P<0.01)$ (Table 5). Total AVLT score was positively correlated with the scores of TDI, THR, DIS and ID $(P<0.05)$ (Supplemental table 2).

The score of CFT-delayed in the AD-OD group was decreased compared with the AD-NOD group $(P<0.01)$ (Table 5). CFT-delayed score was positively correlated with the scores of TDI, THR and DIS $(P<0.01)$ (Supplemental table 2).

There was no difference in AFT score between the AD-OD and AD-NOD groups $(P>0.05)$ (Table 5).

The score of SDMT in the AD-OD group was significantly lower than that in the AD-NOD group $(P<0.01)$ (Table 5). SDMT score was positively correlated with the scores of TDI, DIS and ID $(P<0.05)$ (Supplemental table 3).

The time used for TMT-A in the AD-OD group was significantly prolonged compared with the AD-NOD group $(P<0.01)$ (Table 5). Time spend for TMT-A was negatively correlated with the scores of TDI, DIS and ID $(P<0.01)$ (Supplemental table 3).

The score of CFT-imitated in the AD-OD group was lower compared with the AD-NOD group $(P<0.01)$ (Table 5). CFT-imitated score was positively correlated with the scores of TDI and DIS $(P<0.01)$ (Supplemental table 3).

There was no difference in the time consumed for SCWT or TMT-B or the score of SCWT between the ADOD and AD-NOD groups $(P>0.05)$ (Table 5). 
Table 6. Comparison of neuropsychiatric symptoms between AD-NOD and AD-OD groups.

\begin{tabular}{llll}
\hline Neuropsychiatric variables & $\begin{array}{c}\text { AD-NOD group } \\
\text { (53 cases) }\end{array}$ & $\begin{array}{c}\text { AD-OD group } \\
\text { (44 cases) }\end{array}$ & $\boldsymbol{P}$ \\
\hline NPI [points, median(quartile)] & $1.00(0.00,2.00)$ & $1.00(0.00,4.00)$ & 0.808 \\
HAMD [points, median(quartile)] & $5.00(2.00,11.50)$ & $4.00(1.00,12.00)$ & 0.816 \\
HAMA [points, median(quartile)] & $4.00(1.00,10.50)$ & $3.50(1.00,10.25)$ & 0.807 \\
CMAI [points, median(quartile)] & $29.00(29.00,29.00)$ & $29.00(29.00,33.00)$ & 0.052 \\
MAES [points, median(quartile)] & $9.50(2.00,17.00)$ & $16.50(9.00,26.00)$ & $\mathbf{0 . 0 2 7}^{*}$ \\
\hline
\end{tabular}

*: P<0.05. NPI: Neuropsychiatric Inventory, HAMD: Hamilton Depression Scale, HAMA: Hamilton Anxiety Scale, CMAI: Cohen-

Mansfield Agitation Inventory, MAES: Modified Apathy Evaluation Scale

(2) Relationships between the scores of olfactory function and neuropsychiatric symptoms of AD

MAES score in the AD-OD group was significantly elevated compared with the AD-NOD group $(P<0.05)$ (Table 6). MAES score was negatively correlated with the scores of TDI, DIS and ID $(P<0.05)$ (Supplemental Table 4).

CMAI score in the AD-OD group rose compared with the AD-NOD group, which difference was close to statistical significance $(P=0.052)$ (Table 6$)$.

There was no difference in NPI number, HAMD score, or HAMA score between the AD-OD group and AD-NOD group $(P>0.05)$ (Table 6$)$.

(3) The relationships between the scores of olfactory function and $\mathrm{ADL}$ of $\mathrm{AD}$

ADL score in the AD-OD group was higher than that in the AD-NOD group $(P<0.01)$. ADL score was negatively correlated with the scores of TDI, THR, DIS and ID $(P<0.01)$ (Supplemental table 5).

\section{DISCUSSION}

Olfactory function is vulnerable to multiple factors. For example, the majority of AD patients are elderly, so they are not very sensitive to the alteration of olfactory function. In addition, the decline of olfactory function may not be evident at the early stage of AD. Hence, it is easily ignored by both patients and doctors.

A number of risk factors for AD have been identified, among which age is the most obvious [23]. In this study, the AD dementia group had older age and age of onset than the MCI due to AD group (Table 1). These data suggest that aging is involved in AD dementia. In the 2011 NIA-AA diagnostic criteria [14], AD was regarded as a continuous disease process, including stages of asymptomatic preclinical $\mathrm{AD}, \mathrm{MCI}$ due to $\mathrm{AD}$ and $\mathrm{AD}$ dementia. Accordingly, the age and age of onset of patients with $\mathrm{AD}$ dementia might be older than that of patients with $\mathrm{MCI}$ due to $\mathrm{AD}$.

In this study, OD was evaluated by multiple approaches. First, all subjects reported OD by themselves.
The data showed that the frequency of OD in the control, $\mathrm{MCI}$ due to $\mathrm{AD}$ and $\mathrm{AD}$ dementia groups by self-report was $10.0 \%, 13.5 \%$ and $18.3 \%$, respectively (Table 2), indicating that olfactory function was increasingly impaired as cognitive function deteriorated. However, $100 \%, 80 \%$ and $27.3 \%$ of patients in the control, MCI due to $\mathrm{AD}$ and $\mathrm{AD}$ dementia groups, respectively, wrongly reported OD as demonstrated by the Sniffin' Sticks test, implying a relatively low accuracy of self-report in OD evaluation, especially in the control and MCI due to $\mathrm{AD}$ groups. Hence, OD reported by the two groups needed further validation by using other olfactory tests. Doty. et al [3] reported that only $6 \%$ of AD patients had complaints of OD in the early stage of disease, while $90 \%$ of AD patients actually had impairment of olfactory function as demonstrated by olfactory tests. Therefore, it is necessary to detect olfactory function by using sensitive and objective tests, particularly in the early stage of $\mathrm{AD}$, to more accurately identify AD patients with OD.

Second, HRS was used for screening OD. The frequency of $\mathrm{OD}$ also increased from the control group to the $\mathrm{MCI}$ due to $\mathrm{AD}$ group to the $\mathrm{AD}$ dementia group (Table 2), illustrating that olfactory function was progressively impaired as cognitive function deteriorated. Here, in the MCI due to AD group, 9 cases (24.3\%) had OD by HRS. Compared with the results from the Sniffin' Sticks test, HRS had higher sensitivity, specificity, negative predictive value and diagnostic coincidence rate. These results suggest that HRS might serve as a valuable tool of screening OD for MCI due to AD patients for its ease, convenience, reliability and time-saving. In the $\mathrm{AD}$ dementia group, 29 patients $(48.3 \%)$ had OD by HRS. The sensitivity, specificity, negative predictive value and diagnostic coincidence rate of HRS in the AD dementia group were all lower than in the MCI due to AD group, indicating that HRS might not be suitable for $\mathrm{AD}$ dementia patients.

Third, the Sniffin' Sticks test was adopted to objectively and comprehensively evaluate olfactory function. The data showed that the frequency of OD increased in the control, MCI due to AD and AD dementia groups as above (Table 2), suggesting that olfactory 
function was increasingly damaged as cognitive function declined. In the control group, the frequency of OD by the Sniffin' Sticks test was lower than that by both self-report and HRS. We speculate that normal subjects might be more sensitive to olfaction, but the frequency of OD was not high when HRS was used. The frequency of OD in this group by the Sniffin' Sticks test was the lowest because this test was more objective and comprehensive. In the MCI due to AD group, the frequency of OD by the Sniffin' Sticks test was lower than that by HRS. However, in the AD dementia group, the frequency of OD by the Sniffin' Sticks was higher than that by HRS and selfreport, suggesting that the Sniffin' Sticks test was more suitable for $\mathrm{OD}$ evaluation in patients with $\mathrm{AD}$ dementia.

Of the 97 AD patients, $44=(45.4 \%)$ had OD by the Sniffin' Sticks test, indicating that OD was a common non-cognitive symptom of AD. Comparing the above three methods of olfactory evaluation, the Sniffin' Sticks test was able to find = more AD patients with OD, which might be attributed to its comprehensive actions of assessing olfaction in multiple aspects.

In this study, compared with the control group, olfactory threshold and discrimination of the MCI due to AD group was significantly impaired (Table 3). Compared with the MCI due to $\mathrm{AD}$ group, olfactory threshold, discrimination and identification of the AD dementia group were all significantly decreased (Table 3 ), even after adjusting age and age of onset. The above data further illustrate that the more severe the cognitive impairment, the worse the olfactory dysfunction.

In this study, the scores of TDI, THR, DIS and ID in the AD-OD group were all significantly decreased compared to the AD-NOD group (Table 4), illustrating that $\mathrm{AD}-\mathrm{OD}$ was characterized by an overall decline of olfactory threshold, discrimination and identification.

The impaired cognitive domains of $\mathrm{AD}$ include memory, attention, language, visuospatial ability and executive function. The MMSE and MOCA scales cover the above cognitive domains and are commonly used for evaluating overall cognitive impairment. In this study, compared with the AD-NOD group, the overall cognitive function of the AD-OD group was significantly impaired, as indicated by the decreased scores of MMSE and MoCA scales (Table 5). Furthermore, the scores of the two scales were both positively correlated with the scores of TDI, THR, DIS and ID (Supplemental table 1), showing that olfactory function was increasingly impaired as cognitive level declined in $\mathrm{AD}$ patients.

A previous investigation reported an association between olfactory identification and cognition in AD patients by using MMSE and UPSIT for evaluation of global cognitive function and olfactory identification, respectively [2]. In the current study, in contrast to the above investigation, in addition to olfactory identification, olfactory threshold and discrimination by the Sniffin' Sticks test were also associated with overall cognitive function as rated by MMSE and MoCA, suggesting that the Sniffin' Sticks test has advantages for investigating overall OD and its relationship with cognitive impairment in $\mathrm{AD}$ patients.

In this study, AVLT and CFT were used to rate verbal memory and visual delayed memory, respectively. The AD-OD group had more seriously impaired overall memory and delayed memory than the AD-NOD group (Table 5). Moreover, the overall memory and delayed memory drastically deteriorated, as total olfactory function and olfactory threshold, identification and discrimination were all evidently impaired (Supplemental table 2). Additionally, visual delayed memory declined with compromised overall olfactory function, olfactory threshold and discrimination (Supplemental table 2). A recent meta-analysis [24] suggested that olfactory identification and discrimination were impaired in $\mathrm{AD}$ patients, indicating that high-level olfactory tasks involved specific cognitive processes, while olfactory threshold rather relied on low-level perceptual processes $[9,25]$. In contrast to the results from other researchers reporting that olfactory identification could predict memory decline [26], the current study indicates that overall OD and impairments of olfactory threshold, identification and discrimination all might predict the decline of memory, particularly delay-memory, which might be because the Sniffin' Sticks test evaluates olfactory function comprehensively while UPSIT, used by most of other investigators, only assesses olfactory identification.

In the present study, SMDT and TMT-A were used to assess attention, including visual scanning, attention segmentation, tracking and motion speed [20]. The ADOD group had significantly poorer attention than the ADNOD group (Table 5). In addition, attention was further compromised when olfactory identification and discrimination were increasingly declined (Supplemental table 3). Deficit in attention might occur at earlier stages of AD [27], and attention might be the second impaired cognitive domain followed by memory, earlier than language and visuospatial ability [28]. Olfaction and advanced cognition have common brain regions, such as the hippocampus, amygdala, temporal lobe. The brain regions activated by attention overlap with the olfactionrelated ones, which might be the common anatomical basis for OD and attention deficit in AD patients [29]. The olfactory function of the AD-OD group was significantly impaired in the current investigation, and more importantly, the attention deficit of $\mathrm{AD}$ patients was related to olfactory identification and discrimination. Therefore, we hypothesize that $\mathrm{AD}$ patients have to concentrate their attention to experience and remember 
every odorant in the process of olfactory identification and discrimination tests. Hence, attention deficit influenced the process of identification and discrimination of $\mathrm{AD}$ patients.

CFT-imitation was used to evaluate visuospatial ability [17]. Our data imply that visuospatial ability in the $\mathrm{AD}-\mathrm{OD}$ group was impaired compared with the AD-NOD group (Table 5). Moreover, with the impairment of visuospatial ability, the olfactory discrimination of $\mathrm{AD}$ patients was significantly declined (Supplemental table $3)$. Visuospatial impairment is one of the prominent symptoms of $\mathrm{AD}$ patients, and it even appears in the MCI stage [30]. Two pathways are responsible for visuospatial ability. The ventral pathway, originating from the occipital lobe and projecting to the lower temporal cortex, is mainly in charge of perception, identification of objects seen by the naked eye and the storage of spatial memory in the medial temporal lobe and hippocampus. The dorsal pathway, originating from the occipital lobe and projecting to the parietal lobe, prefrontal cortex, premotor cortex and medial temporal lobe, is mainly in charge of formation of visuospatial memory, visual navigation and subsequent processing of visuospatial ability [31]. Moreover, the olfaction-related brain regions include the medial temporal lobe and hippocampus. Therefore, we hypothesize that olfactory function and visuospatial ability share a common anatomical basis.

Apathy is a prevalent neuropsychiatric manifestation of $\mathrm{AD}$, which leads to a compromised daily function and increased burden of caregivers [32]. A previous study reported a specific association between olfactory identification performance and apathy severity, suggesting that olfactory dysfunction and apathy might result from the progression of disease pathology in shared neural substrates [33]. In this study, the AD-OD group was more likely to suffer from apathy than the AD-NOD group (Table 6). With the aggravation of apathy, the function of olfactory identification and discrimination were increasingly impaired (Supplemental table 4). The apathy of $\mathrm{AD}$ patients was associated with olfactory discrimination. The core of apathy is a lack of motivation for emotion, cognition and behavior, which might affect the desire to participate in the tests of odor identification and discrimination. Therefore, dysfunction of olfactory discrimination of $\mathrm{AD}$ might be related to apathy.

The present investigation showed that the CMAI score of the AD-OD group was higher than that of the ADNOD group, and the difference was close to statistical significance (Table 6), illustrating that the AD-OD group might be more prone to agitation than the AD-NOD group. The agitation in MCI due to $\mathrm{AD}$ patients and $\mathrm{AD}$ dementia patients is related to atrophy of frontal lobe, insular lobe, amygdala, cingulate gyrus and hippocampus [34], and coincidently, the insular lobe, amygdala and hippocampus are also olfaction-related brain regions, indicating $\mathrm{OD}$ and agitation share common neuroanatomical basis.

This study revealed that OD dramatically impaired the ADL of AD patients. We speculate that the damage to global cognitive function and multiple cognitive domains of memory, visuospatial ability and attention together with severer apathy amplified the negative impact on $\mathrm{ADL}$ of patients in the AD-OD group.

In summary, the frequency and accuracy of OD by self-report is relatively low. HRS can be used for screening olfaction in patients with MCI due to AD. The Sniffin' Sticks test can be used for validating OD in AD patients. OD is prevalent in $\mathrm{AD}$ patients, as evidenced by the overall declines of olfactory threshold, discrimination and identification. AD-OD patients have severe impairments in global cognition and multiple cognitive domains of memory, visuospatial ability and attention, as well as neuropsychiatric symptoms of apathy, and thus have seriously compromised ADL.

\section{Acknowledgments}

This work was supported by the National Key Research and Development Program of China (2016YFC1306300, 2016YFC1306000), the National Natural Science Foundation of China (81571229, 81071015, 30770745), the Key Project of the National Natural Science Foundation of China (81030062), the Key Project of the Natural Science Foundation of Beijing, China (B) (kz201610025030), the Key Project of the Natural Science Foundation of Beijing, China (4161004, kz200910025001), the Natural Science Foundation of Beijing, China (7082032), the National Key Basic Research Program of China (2011CB504100), the Important National Science and Technology Specific Projects (2011ZX09102-003-01), the National Key Technology Research and Development Program of the Ministry of Science and Technology of China (2013BAI09B03), the Project of Beijing Institute for Brain Disorders (BIBD-PXM2013_014226_07_000084), the High Level Technical Personnel Training Project of Beijing Health System, China (2009-3-26), the Project of Construction of Innovative Teams and Teacher Career Development for Universities and Colleges Under Beijing Municipality (IDHT20140514), Capital Clinical Characteristic Application Research (Z12110700100000, Z121107001012161), the Beijing Healthcare Research Project, China (JING-15-2, JING-15-3), the Excellent Personnel Training Project of Beijing, China (20071D0300400076, 2016000021469G209), BasicClinical Research Cooperation Funding of Capital Medical University, China (2015-JL-PT-X04, 10JL49, 14JL15), and Youth Research Funding, Beijing Tiantan 
Hospital, Capital Medical University, China (2014-YQNYS-18, 2015-YQN-15, 2015-YQN-05, 2015-YQN-14, 2015-YQN-17).

\section{Conflict of interest}

The authors declare that they have no competing interests.

\section{Supplementary data}

Supplementary data is available online at www.aginganddisease.org/EN/10.14336/AD.2018.0819

\section{References}

[1] Mesholam RI, Moberg PJ, Mahr RN, Doty RL (1998). Olfaction in neurodegenerative disease: a meta-analysis of olfactory functioning in Alzheimer's and Parkinson's diseases. Arch Neurol, 55:84-90.

[2] Velayudhan L, Pritchard M, Powell JF, Proitsi P, Lovestone S (2013). Smell identification function as a severity and progression marker in Alzheimer's disease. Int Psychogeriatr, 25:1157-1166.

[3] Doty RL, Reyes PF, Gregor T (1987). Presence of both odor identification and detection deficits in Alzheimer's disease. Brain Res Bull, 18:597-600.

[4] Devanand DP, Tabert MH, Cuasay K, et al. ( 2010). Olfactory identification deficits and MCI in a multiethnic elderly community sample. Neurobiol Aging, 31:1593-1600.

[5] Velayudhan L (2015). Smell identification function and Alzheimer's disease: a selective review. Curr Opin Psychiatry, 28:173-179.

[6] Djordjevic J, Jones-Gotman M, De Sousa K, Chertkow $\mathrm{H}$ (2008). Olfaction in patients with mild cognitive impairment and Alzheimer's disease. Neurobiol Aging, 29:693-706.

[7] Doty RL, Shaman P, Kimmelman CP, Dann MS (1984). University of Pennsylvania Smell Identification Test: a rapid quantitative olfactory function test for the clinic. Laryngoscope, 94:176-178.

[8] Hummel T, Kobal G, Gudziol H, Mackay-Sim A (2007). Normative data for the "Sniffin' Sticks" including tests of odor identification, odor discrimination, and olfactory thresholds: an upgrade based on a group of more than 3,000 subjects. Eur Arch Otorhinolaryngol, 264:237-243.

[9] Barresi M, Ciurleo R, Giacoppo S, et al. (2012). Evaluation of olfactory dysfunction in neurodegenerative diseases. J Neurol Sci, 323:16-24.

[10] Sohrabi HR, Bates KA, Weinborn MG, et al. (2012). Olfactory discrimination predicts cognitive decline among community-dwelling older adults. Transl Psychiatry, 2:e118.

[11] Devanand DP, Lee S, Manly J, et al.( 2015). Olfactory deficits predict cognitive decline and Alzheimer dementia in an urban community. Neurology, 84:182189.
[12] Hedner M, Larsson M, Arnold N, Zucco GM, Hummel T (2010). Cognitive factors in odor detection, odor discrimination, and odor identification tasks. J Clin Exp Neuropsychol, 32:1062-1067.

[13] Albert MS, DeKosky ST, Dickson D, et al. (2011). The diagnosis of mild cognitive impairment due to Alzheimer's disease: recommendations from the National Institute on Aging-Alzheimer's Association workgroups on diagnostic guidelines for Alzheimer's disease. Alzheimers Dement, 7:270-279.

[14] McKhann GM, Knopman DS, Chertkow H, et al. (2011). The diagnosis of dementia due to Alzheimer's disease: recommendations from the National Institute on AgingAlzheimer's Association workgroups on diagnostic guidelines for Alzheimer's disease. Alzheimers Dement, 7:263-269.

[15] Millar Vernetti P, Perez Lloret S, Rossi M, Cerquetti D, Merello M (2012). Validation of a new scale to assess olfactory dysfunction in patients with Parkinson's disease. Parkinsonism Relat Disord, 18:358-361.

[16] Guo Q, Zhao Q, Chen M, Ding D, Hong Z (2009). A comparison study of mild cognitive impairment with 3 memory tests among Chinese individuals. Alzheimer Dis Assoc Disord, 23:253-259.

[17] Zhou Y LJ, Guo QH, Hong ZN. (2006). Rey-Osterrieth complex figure test used to identify mild Alzheimer's disease. Chin J Clin Neurosci, 14:501-504.

[18] Lin CY, Chen TB, Lin KN, et al. (2014). Confrontation naming errors in Alzheimer's disease. Dement Geriatr Cogn Disord, 37:86-94.

[19] Guo QH SY YJ, Hong Z, Lu CZ (2007). Application of eight executive tests in participants at Shanghai communities. Chin J Behav Med Sci, 16:628-631.

[20] YX G (1992). Wechsler adult intelligence scale-chinese version(WAIS). Hunan: Hunan Map Press.

[21] Lawton MP, Brody EM (1969). Assessment of older people: self-maintaining and instrumental activities of daily living. Gerontologist, 9:179-186.

[22] Katz S, Ford AB, Moskowitz RW, Jackson BA, Jaffe MW (1963). Studies of Illness in the Aged. The Index of ADL: A Standardized Measure of Biological and Psychosocial Function. JAMA, 185:914-919.

[23] Murphy C, Solomon ES, Haase L, Wang M, Morgan CD (2009). Olfaction in aging and Alzheimer's disease: event-related potentials to a cross-modal odorrecognition memory task discriminate ApoE epsilon4+ and ApoE epsilon 4- individuals. Ann N Y Acad Sci, 1170:647-657.

[24] Rahayel S, Frasnelli J, Joubert S (2012). The effect of Alzheimer's disease and Parkinson's disease on olfaction: a meta-analysis. Behav Brain Res, 231:60-74.

[25] Larsson M, Nilsson LG, Olofsson JK, Nordin S (2004). Demographic and cognitive predictors of cued odor identification: evidence from a population-based study. Chem Senses, 29:547-554.

[26] Swan GE, Carmelli D (2002). Impaired olfaction predicts cognitive decline in nondemented older adults. Neuroepidemiology, 21:58-67.

[27] Perry RJ, Watson P, Hodges JR (2000). The nature and staging of attention dysfunction in early (minimal and 
mild) Alzheimer's disease: relationship to episodic and semantic memory impairment. Neuropsychologia, 38:252-271.

[28] Grady CL, Haxby JV, Horwitz B, et al.( 1988). Longitudinal study of the early neuropsychological and cerebral metabolic changes in dementia of the Alzheimer type. J Clin Exp Neuropsychol. 10:576-596.

[29] Levy LM, Henkin RI, Hutter A, Lin CS, Martins D, Schellinger D (1997). Functional MRI of human olfaction. J Comput Assist Tomogr, 21:849-856.

[30] Tales A, Haworth J, Nelson S, Snowden RJ, Wilcock G (2005). Abnormal visual search in mild cognitive impairment and Alzheimer's disease. Neurocase, 11:8084.
[31] Watson CE, Chatterjee A (2012). A bilateral frontoparietal network underlies visuospatial analogical reasoning. Neuroimage, 59:2831-2838.

[32] Landes AM, Sperry SD, Strauss ME, Geldmacher DS (2001). Apathy in Alzheimer's disease. J Am Geriatr Soc, 49:1700-1707.

[33] Seligman SC, Kamath V, Giovannetti T, Arnold SE, Moberg PJ (2013). Olfaction and apathy in Alzheimer's disease, mild cognitive impairment, and healthy older adults. Aging Ment Health, 17:564-570.

[34] Trzepacz PT, Yu P, Bhamidipati PK, et al. (2013). Frontolimbic atrophy is associated with agitation and aggression in mild cognitive impairment and Alzheimer's disease. Alzheimers Dement, 9:S95-S104.e101. 